\title{
ALPPS for Locally Advanced Intrahepatic Cholangiocarcinoma: Did Aggressive Surgery Lead to the Oncological Benefit? An International Multi-center Study
}

Jun Li, MD ${ }^{1}$ (D), Mohamed Moustafa, MD, $\mathrm{PhD}^{2}$, Michael Linecker, MD, $\mathrm{PhD}^{3}$, Georg Lurje, $\mathrm{MD}^{4}$, Ivan Capobianco, $\mathrm{MD}^{5}$, Janine Baumgart, $\mathrm{MD}^{6}$, Francesca Ratti, $\mathrm{MD}^{7}$, Falk Rauchfuss, $\mathrm{MD}, \mathrm{MSc}^{8}$, Deniz Balci, MD ${ }^{9}$, Eduardo Fernandes, $\mathrm{MD}^{10,11}$, Roberto Montalti, $\mathrm{MD}^{12}$, Ricardo Robles-Campos, $\mathrm{MD}$, PhD ${ }^{13}$, Bergthor Bjornsson, MD, $\mathrm{PhD}^{14}$, Stefan A. Topp, $\mathrm{MD}^{15}$, Jiri Fronek, MD, PhD ${ }^{16,17}$, Chao Liu, $\mathbf{M D}^{18}$, Roger Wahba, MD, PhD ${ }^{19}$, Christiane Bruns, MD, $\mathbf{P h D}^{19}$, Stefan M. Brunner, $\mathrm{MD}^{20}$, Hans J. Schlitt, $\mathbf{M D}^{20}$, Asmus Heumann, MD ${ }^{1}$, Björn-Ole Stüben, MD ${ }^{1}$, Jakob R. Izbicki, MD ${ }^{1}$, Jan Bednarsch, $\mathrm{MD}^{4}$, Enrico Gringeri, $\mathrm{MD}^{2}$, Elisa Fasolo ${ }^{2}$, Jens Rolinger, $\mathrm{MD}^{5}$, Jakub Kristek, MD ${ }^{16,17}$, Roberto Hernandez-Alejandro, $\mathrm{MD}^{21}$, Andreas Schnitzbauer, $\mathrm{MD}^{22}$, Natascha Nuessler, $\mathrm{MD}^{23}$, Michael R. Schön, $\mathrm{MD}^{24}$,

Sergey Voskanyan, MD, PhD $^{25}$, Athanasios S. Petrou, $\mathbf{M D}^{26}$, Oszkar Hahn, MD, $\mathbf{P h D}^{27}$, Yuji Soejima, MD, $\mathbf{P h D}^{28}$, Emilio Vicente, $\mathrm{MD}, \mathrm{PhD}^{29}$, Carlos Castro-Benitez, $\mathrm{MD}, \mathbf{P h D}^{30}$, René Adam, $\mathbf{M D}, \mathbf{P h D}^{30}$, Federico Tomassini, $\mathrm{MD}^{31}$, Roberto Ivan Troisi, $\mathrm{MD}^{31,32}$, Alexandros Kantas, $\mathrm{MD}^{33}$, Karl Juergen Oldhafer, MD, $\mathbf{P h D}^{33}$, Victoria Ardiles, $\mathrm{MD}, \mathrm{PhD}^{34}$, Eduardo de Santibanes, $\mathrm{MD}, \mathrm{PhD}^{34}$, Massimo Malago, $\mathbf{M D}^{35}$, Pierre-Alain Clavien, MD, $\mathrm{PhD}^{3}$, Marco Vivarelli, $\mathrm{MD}^{36}$, Utz Settmacher, $\mathrm{MD}^{8}$, Luca Aldrighetti, $\mathrm{MD}^{7}$, Ulf Neumann, $\mathrm{MD}^{4}$, Henrik Petrowsky, $\mathrm{MD}^{3}$, Umberto Cillo, $\mathrm{MD}, \mathrm{PhD}^{2}$, Hauke Lang, $\mathrm{MD}^{6}$, and Silvio Nadalin, $\mathrm{MD}^{5}$

${ }^{1}$ Department of General, Visceral and Thoracic Surgery, University Medical Center Hamburg-Eppendorf, Hamburg, Germany; ${ }^{2}$ Hepatobiliary Surgery and Liver Transplant Unit, University of Padua, Padua, Italy; ${ }^{3}$ Swiss HPB and Transplantation Center, Department of Surgery and Transplantation, University Hospital Zurich, Zurich, Switzerland; ${ }^{4}$ Department of Surgery and Transplantation, University Hospital RWTH Aachen, Aachen, Germany; ${ }^{5}$ Department of General, Visceral and Transplantation Surgery, University Hospital Tuebingen, Tübingen, Germany; ${ }^{6}$ Department of General, Visceral and Transplantation Surgery, University Hospital Mainz, Mainz, Germany; ${ }^{7}$ Hepatobiliary Surgery Division, San Raffaele Hospital, Milan, Italy; ${ }^{8}$ Department of General, Visceral and Vascular Surgery, University Hospital Jena, Jena, Germany; ${ }^{9}$ Department of Surgery, Ankara University, Ankara, Turkey; ${ }^{10}$ Department of Surgery, Federal University of Rio de Janeiro, Rio de Janeiro, Brazil; ${ }^{11}$ Department of Surgery and Transplantation, São Lucas Hospital Copacabana, Rio de Janeiro, Brazil; ${ }^{12}$ Department of Public Health, Federico II University Hospital, Naples, Italy;

${ }^{13}$ Department of Surgery, Virgen de la Arrixaca Hospital, IMIB-Arrixaca, Murcia, Spain; ${ }^{14}$ Department of Surgery and Department of Clinical and Experimental Medicine, Linköping University, Linköping, Sweden; ${ }^{15}$ Department of General, Visceral and Pediatric Surgery, University Hospital Düsseldorf, Düsseldorf, Germany; ${ }^{16}$ Department of Transplant Surgery, Institute for Clinical and Experimental Medicine, Prague, Czech Republic; ${ }^{17}$ Department of Anatomy, Second Faculty of Medicine, Charles University, Prague, Czech Republic; ${ }^{18}$ Department of Hepato-Pancreato-Biliary Surgery, Sun Yat-Sen Memorial Hospital, Sun Yat-Sen University, Guangzhou, China; ${ }^{19}$ Department of General, Visceral, Cancer and

The paper is based on the oral presentation at the 13th Congress of the E-AHPBA, June 2019 (Abstract number 294).

$\mathrm{HL}$ and $\mathrm{SN}$ have contributed equally to this work.

(C) The Author(s) 2020

First Received: 17 October 2019;

Published Online: 30 January 2020

J. Li, MD

e-mail: j.li@uke.de 
Transplantation Surgery, University Hospital of Cologne, Cologne, Germany; ${ }^{20}$ Department of Surgery, University Medical Center Regensburg, Regensburg, Germany; ${ }^{21}$ Division of Transplantation and Hepatobiliary Surgery, University of Rochester, Rochester, NY; ${ }^{22}$ Department of General, Visceral and Transplantation Surgery, University Hospital Frankfurt, Frankfurt, Germany; ${ }^{23}$ Department of General, Visceral and endocrine Surgery, München Klinik Neuperlach, Munich, Germany; ${ }^{24}$ Klinikum Karlsruhe, Karlsruhe, Germany; ${ }^{25}$ Center for Surgery and Transplantology, A.I. Burnazyan Russian State Scientific Center FMBC of FMBA, Moscow, Russia; ${ }^{26}$ Department of General Surgery, Nicosia Teaching Hospital, Strovolos, Cyprus; ${ }^{27} 1$ st Department of Surgery, Semmelweis University, Budapest, Hungary; ${ }^{28}$ Department of Surgery, Shinshu University School of Medicine, Matsumoto, Japan; ${ }^{29}$ "Clara Campal" Oncological Center, Sanchinarro University Hospital, San Pablo University. CEU, Madrid, Spain; ${ }^{30}$ Centre Hépato-Biliaire, AP-HP Hôpital Paul Brousse, Inserm U 935, Univ Paris-Saclay, Villejuif, France; ${ }^{31}$ Department of Human Structure and Repair, Faculty of Medicine, Ghent University, Ghent, Belgium; ${ }^{32}$ Department of Clinical Medicine and Surgery, Federico II University, Naples, Italy;

${ }^{33}$ Department of Surgery, Division of HPB Surgery, Asklepios Hospital Barmbek, Semmelweis University Budapest, Campus Hamburg, Hamburg, Germany; ${ }^{34}$ HPB Surgery and Liver Transplant Unit, Italian Hospital Buenos Aires, Buenos Aires, Argentina; ${ }^{35}$ Department of Surgery, University College London, London, UK; ${ }^{36}$ Hepatobiliary and Abdominal Transplantation Surgery, Department of Experimental and Clinical Medicine, Polytechnic University of Marche, Ancona, Italy

\section{ABSTRACT}

Background. ALPPS is found to increase the resectability of primary and secondary liver malignancy at the advanced stage. The aim of the study was to verify the surgical and oncological outcome of ALPPS for intrahepatic cholangiocarcinoma (ICC).

Methods. The study cohort was based on the ALPPS registry with patients from 31 international centers between August 2009 and January 2018. Propensity score matched patients receiving chemotherapy only were selected from the SEER database as controls for the survival analysis.

Results. One hundred and two patients undergoing ALPPS were recruited, 99 completed the second stage with median inter-stage duration of 11 days. The median kinetic growth rate was $23 \mathrm{ml} /$ day. $\mathrm{R} 0$ resection was achieved in 87 $(85 \%)$. Initially high rates of morbidity and mortality decreased steadily to a $29 \%$ severe complication rate and $7 \%$ 90-day morbidity in the last 2 years. Post-hepatectomy liver failure remained the main cause of 90-day mortality. Multivariate analysis revealed insufficient future liver remnant at the stage-2 operation (FLR2) to be the only risk factor for severe complications (OR 2.91, $p=0.02$ ). The propensity score matching analysis showed a superior overall survival in the ALPPS group compared to palliative chemotherapy (median overall survival: 26.4 months vs 14 months; 1-, 2-, and 3-year survival rates: $82.4 \%, 70.5 \%$ and $39.6 \%$ vs $51.2 \%, 21.4 \%$ and $11.3 \%$, respectively, $p<0.01$ ). The survival benefit, however, was not confirmed in the subgroup analysis for patients with insufficient FLR2 or multifocal ICC.

Conclusion. ALPPS showed high efficacy in achieving R0 resections in locally advanced ICC. To get the most oncological benefit from this aggressive surgery, ALPPS would be restricted to patients with single lesions and sufficient FLR2.

Intrahepatic cholangiocarcinoma (ICC) is the second most common primary liver tumor and its incidence is increasing worldwide. ${ }^{1-3}$ Liver resection (LR), mostly major hepatectomy, is the gold standard treatment with curative intention. ${ }^{4-6}$ For patients presenting with unresectable ICC, systemic chemotherapy remains the mainstay palliative treatment modality without long-term survival. ${ }^{7,8}$ Five-year overall survival after liver resection has been reported in the range $22-44 \%,{ }^{9}$ whilst approximately $58 \%$ present with tumor recurrence within 24 months. ${ }^{10}$ To judge the resectability, sufficient future liver remnant (FLR) is a main factor beside the possibility of vascular as well as biliary resections and reconstructions. ${ }^{11-13}$

In order to avoid post-hepatectomy liver failure (PHLF), various procedures, including two-stage hepatectomy and portal vein ligation known as associating liver partition and portal vein ligation for staged hepatectomy (ALPPS), have been performed to induce hypertrophy of the FLR. ALPPS was first reported by Schnitzbauer et al. in 2012. ${ }^{14,15}$ This innovative technique was rapidly adopted by hepatobiliary centers in the management of advanced liver tumors due to its promising high R0 resection rate. ${ }^{16-19}$ Nevertheless, some legitimate concerns were raised due to its high morbidity and mortality in comparison to conventional major hepatectomies. ${ }^{20-23}$ The mid- and long-term oncological outcomes of the ALPPS procedure performed for locally advanced ICC patients remain unverified. The potential oncological benefit 
of this aggressive surgical approach over alternative therapeutic modalities such as chemotherapy has not been investigated to date.

\section{AIM}

The primary objective of this study was to investigate the oncological benefit of the ALPPS procedure for locally advanced ICC in comparison to chemotherapy. Knowing that ALPPS is associated with high morbidity and early mortality, identifying the risk factors as well as the subgroup of patients that might not benefit from this procedure was the secondary objective.

\section{PATIENTS AND METHODS}

\section{Study Design}

The present cohort was composed of data derived from the International ALPPS Registry (ClinicalTrials.gov: NCT01924741). The study was approved by the Scientific Committee of the ALPPS Registry on June 18, 2017 (http:// www.alpps.net/?q=node/88). Other hepatobiliary centers that were not on the registry at that time were encouraged to register themselves. Questionnaires were sent to all centers to complete the items required, especially perioperative outcome as well as long-term outcome of the ALPPS procedure for ICC patients. Since patients undergoing ALPPS were those usually regarded as unresectable and would have had palliative chemotherapy, a group of patients receiving only palliative chemotherapy in the Surveillance, Epidemiology, and End Results Program (SEER) database (https://seer.cancer.gov) was chosen as a comparison to study the oncological benefits of the ALPPS procedure.

The study consisted of three main parts: (1) analyzing the safety and efficacy (in terms of resectability) of ALPPS in a multi-centric database and identifying the risk factors for postoperative morbidity and 90-day mortality; (2) comparing the overall survival with a propensity-score matched group of patients who received palliative chemotherapy from a national database; and (3) a supplementary analysis was performed to identify a subgroup of patients who might not benefit from the ALPPS procedure.

\section{Study Population}

The registry data of ALPPS was first exported for the latest analysis on November 11, 2017. All centers updated the information until May 22, 2019. Patients with primary ICC who received chemotherapy without surgical resection were retrieved from the SEER database.

\section{Variables Definition}

For the ALPPS group, data on patient demographics, comorbidities, reason for performing ALPPS, volumetric data, procedure details, postoperative complications, liver parenchyma status, tumor pathology, and follow-up with details on recurrence and survival status were provided by the ALPPS registry as well as by the participating centers.

For the chemotherapy group, the SEER * Stat statistical software (version 8.3.2) was utilized to select the study population. In this version, data of patients classified by the 7th edition of the AJCC TNM staging system were available during the period from January 2010 till December 2013. The primary site code "C22.1" referred to the intrahepatic bile duct, and the ICDO-3 histology/behavior code "8160/3" was for cholangiocarcinoma. The chemotherapy data were labeled as "Yes" vs "No/unknown". Only cases labeled "Yes" for chemotherapy were included in the study. The chemotherapy regimens were not described in detail. Patients with ICC were identified according to the International Classification of Diseases for Oncology, 3rd edition, ICD-O-3/WHO, 2008. The codes for patient demographics, tumor size, multifocality, vascular invasion, lymph node metastases and survival were interpreted using the Collaborative Stage data set (http:// web2.facs.org/cstage0205/liver/Liverschema.html).

\section{Outcome Assessment}

Volumetry study was represented by FLR to standard total liver volume ${ }^{24}$ (FLR/sTLV) ratio and FLR to body weight (FLR/BW) ratio. The median values of FLR/BW and FLR/sTLV after stage-1 and stage-2 hepatectomies were utilized as cut-off values for logistic regression analyses. To standardize kinetic growth, a mean volume ( $\mathrm{ml}$ and \%) increase per day was calculated assuming a linear growth model. ${ }^{25}$ Growth was expressed in FLR increase per day in percent. Complications were identified according to liver surgery specific clinical endpoints (CEP). ${ }^{26,27}$ The five elements of CEP beside mortality are PHLF, ascites, bile leak, infection and post hepatectomy hemorrhage (PHH). Complications were graded according to the definitions of the International Study Group of Liver Surgery (ISGLS) and the Clavien-Dindo classification. ${ }^{28-31}$ We defined severe complications as a Clavien-Dindo grade $3 \mathrm{~b}$ or greater, including postoperative mortality (i.e., grade 5). The histological data consisted of TNM staging according to the 7th edition of AJCC staging system and tumor resection margin status. The follow-up data in the ALPPS group included the survival status, reason for death, recurrence status, time to recurrence, or death. Time to recurrence was defined as being from the stage- 2 operation until recurrence (hepatic or extrahepatic). Overall survival 
(OS) was defined as the date of the first stage operation of ALPPS until death or last follow-up.

In the chemotherapy group the outcome included survival status and cause of death. The survival per month variable in the SEER data is explicitly reported in numbers, which represent the time from diagnosis to death (for deceased patients) or last follow-up (for alive patients). Cause of death was reported as "death due to cancer," "death due to other cause," or "unknown."

\section{Statistical Analysis}

Continuous variables are expressed as median (range) and were analyzed by the Mann-Whitney $U$ test. Categorical-nominal variables are presented as a number (percentage) and were analyzed by the C2 or Fisher's exact tests, as appropriate. All the represented percentages in the result section have excluded the missing values.

For the ALPPS group, uni- and multivariate logistic regression analyses were performed to verify risk factors for severe complications including the 90-day mortality. In order to understand the correlation between risk factors, the phi coefficient was calculated between nominal variables or between nominal variables and modified categorical variables. The Kaplan-Meier method was utilized to calculate overall survival and recurrence, and the log-rank test was used to assess the difference between curves. Cox proportional hazard regression was performed to evaluate risk factors associated with prognosis. Variables with $p<0.1$ in the univariate analysis were further included in the multivariate Cox proportional hazards regression analysis with a stepwise forward conditional selection. Based on the propensity score, one-to-one nearest neighbor matching with replacement was adopted to overcome selection bias and minimize differences between the chemotherapy group and the ALPPS group. The propensity scores calculated by a logistic regression model represent the probability of each patient being assigned to each treatment. The variables included in this model were: age, gender, tumor stage, and lymph node status. Two-tailed $p<0.05$ values were considered statistically significant and all statistical calculations were performed using SPSS version 21.0 (Chicago, IL, USA).

\section{RESULTS}

\section{Demographics and Clinical Features}

A total of 102 patients with ICC undergoing ALPPS in 31 institutes from 18 countries between August 2009 and January 2018 were included. The median number of patients per center was two (range, 1-12). Ten centers performed $\geq 5$ ALPPS for ICC each.

There were 46 men and 56 women, median age 65 years (32-84) (Table 1). The reason for performing ALPPS was insufficient FLR for one-stage hepatectomy according to the surgeon's assessment. In $86.6 \%$ of patients the decision to perform the ALPPS procedure was taken solely according to the liver volume. Insufficient liver function due to diseased liver parenchyma with limited liver volume $^{32}$ was mentioned in $5.2 \%$ of patients. No details were given in the other $8.2 \%$ of patients.

Based on preoperative radiological assessment, a single lesion centrally located was reported in $72.2 \%$. Multiple lesions in the right lobe were reported in $18.6 \%$. Bilobular tumors requiring clearance of FLR was found in $8.8 \%$. Preoperative chemotherapy with gemcitabine and cisplatin was carried out in $7.9 \%$ (Table 1). Preoperative biliary drainage by means of endoscopic retrograde cholangiopancreatography (ERCP) or percutaneous transhepatic cholangiography (PTC) was performed in 5\%.

\section{Procedure Details}

For stage-1 operations intraoperative blood transfusion was reported in $22 \%$ of patients. Lymphadenectomy was performed in $67.4 \%$. Tumor resection in the FLR was reported in $12.2 \%$. Biliary digestive anastomosis was performed in $13.7 \%$. Vascular reconstruction was reported in $2 \%$. Full laparoscopic resection was performed in $6 \%$. Hybrid ALPPS $^{33}$ was performed in $5 \%$. The median intensive care unit (ICU) stay after a stage-1 operation was 1 day (0-30).

In total, 99 patients underwent a stage- 2 operation. The median inter-stage duration was 11 days (3-49). The stage2 operation could not be carried out in three $(2.9 \%)$ patients. Insufficient FLR hypertrophy as well as tumor progression were the reasons reported in two patients. The third patient died due to postoperative hemorrhage after a stage- 1 operation.

In stage-2 operations intraoperative blood transfusion was reported in $33.1 \%$. Right trisectionectomy was performed in $78.7 \%$ of cases. Right hepatectomy was performed in the remaining cases. Biliary digestive anastomosis was performed in $22 \%$. Vascular reconstruction was performed in $12.1 \%$. Full laparoscopic resection was performed in $9.6 \%$. Lymphadenectomy was performed in $20.9 \%$. The median ICU stay after a stage- 2 operation was 1 day (0-43).

\section{Volumetry Study Outcome}

For stage-1 operations the median FLR/sTLV ratio (FLR1/sTLV) was 22\% (9-39\%). The median FLR1/BW 
TABLE 1 Patients' demographic and clinical features

\begin{tabular}{|c|c|c|}
\hline Variable & Definition & $\begin{array}{l}\text { All patients } \\
(n=102)\end{array}$ \\
\hline \multirow[t]{3}{*}{ Age } & & $65(32-84)$ years \\
\hline & $\leq 65$ years & $55(53.9 \%)$ \\
\hline & $>65$ years & $47(46.1 \%)$ \\
\hline \multirow[t]{2}{*}{ Gender } & Female & $56(54.9 \%)$ \\
\hline & Male & $46(45.1 \%)$ \\
\hline BMI & & $25.3(16.3-38.3)$ \\
\hline Diabetes mellitus & Yes & $11(11 \%)$ \\
\hline Other comorbidities & Yes & $26(25.5 \%)$ \\
\hline Preoperative chemotherapy & Yes & $8(7.9 \%)$ \\
\hline \multirow{3}{*}{$\begin{array}{l}\text { Tumor location in preoperative } \\
\text { imaging }\end{array}$} & $1=$ single lesion centrally located & $70(72.2 \%)$ \\
\hline & $\begin{array}{l}2=\text { multiple tumors located in right liver lobe requiring extended right } \\
\text { hepatectomy }\end{array}$ & $18(18.6 \%)$ \\
\hline & $\begin{array}{l}3=\text { Bilobular tumors requiring clearance of FLR and right or extended right } \\
\text { hepatectomy }\end{array}$ & $9(8.8 \%)$ \\
\hline \multirow[t]{3}{*}{ Surgical decision for ALPPS } & $1=$ neither volume nor function of FLR sufficient & $84(86.6 \%)$ \\
\hline & $2=$ volume sufficient but functional FLR insufficient & $5(5.2 \%)$ \\
\hline & $3=$ not specified & $8(8.2 \%)$ \\
\hline FLR stage 1 (FLR1) & & $336(128-664) \mathrm{ml}$ \\
\hline FLR1/sTLV & & $22 \%(9-39 \%)$ \\
\hline FLR1/BW & & $\begin{array}{l}0.46 \% \\
\quad(0.19-0.84 \%)\end{array}$ \\
\hline FLR stage 2 & & $611(270-982) \mathrm{ml}$ \\
\hline FLR2/sTLV & & $40 \%(16-69 \%)$ \\
\hline FLR2/BW & & $\begin{array}{l}0.84 \% \\
\quad(0.35-1.51 \%)\end{array}$ \\
\hline
\end{tabular}

BMI Body mass index, BW Body weight, FLR Future liver remnant, sTLV Standard total liver volume

ratio was $0.46 \%(0.19-0.84 \%)$. For stage- 2 operations the median FLR/sTLV ratio (FLR2/sTLV) was $40 \%$ (16-69\%). The FLR increase was 75\% (range, 3-192\%). The median FLR2/BW ratio was $0.84 \%(0.35-1.51 \%)$. The median kinetic growth rate (KGR) was $7.3 \% /$ day (0.4-24.2\%) or $23 \mathrm{ml} /$ day (2-70). The median KGR was found to be higher in patients with age $<65$ years compared to patients with age $\geq 65$ years $(8.7 \%$ /day vs $6.0 \% /$ day, $p=0.021)$. The quality of liver parenchyma was not confirmed as a factor influencing the KGR $(p=0.426)$.

\section{Postoperative Morbidity and Mortality (Table 2)}

(a) After stage-1 operation:

The overall morbidity after a stage-1 operation was $25 \%$. Liver surgery specific complications were reported as PHLF in $10.8 \%$, ascites in $16.7 \%$, bile leak in $12.6 \%$, infection complications in $13.7 \%$, and $\mathrm{PHH}$ in $26.3 \%$.

Severe complications (Clavien-Dindo grades $3 \mathrm{~b}$ to 5 ) were reported in six patients $(6.1 \%)$. Among them, three were infection complications and the other three were
PHH. No grade C PHLF or bile leak according to the ISGLS classification were reported. One patient died on 13th day after the stage-1 operation due to $\mathrm{PHH}$.

(b) After stage-2 operation:

The overall morbidity after a stage- 2 operation was $76.8 \%$. Liver surgery specific complications were reported as PHLF in $34.9 \%$, ascites in $51.3 \%$, bile leak in $30.9 \%$, infection complications in $43.8 \%$, and $\mathrm{PHH}$ in $31.3 \%$ (Table 2).

Severe complications (Clavien-Dindo grades $3 \mathrm{~b}$ to 5 ) were reported in $41.4 \%$ of the cases, including a 90-day mortality of $21.2 \%$ ( 21 patients). The morbidity and mortality rates steadily decreased over the years (Fig. 1). Among the severe complications, infection complication was reported in $12.5 \%$. Grade C PHLF, bile leak, and PHH were reported in $12.8 \%, 7.2 \%$, and $3.1 \%$, respectively. Therapy refractory ascites (defined as a daily ascites volume more than $1000 \mathrm{ml}$ despite medical treatment after POD 7) developed in $20.3 \%$ patients. 
TABLE 2 Liver surgery-specific complications after stage-1 and stage-2 operation

\begin{tabular}{|c|c|c|c|}
\hline Complications & Definition & $\begin{array}{l}\text { Stage-1 } \\
(n=102)\end{array}$ & $\begin{array}{l}\text { Stage-2 } \\
(n=99)\end{array}$ \\
\hline \multirow[t]{4}{*}{ Liver failure } & No & $74 / 83(89.2 \%)$ & $56 / 86(65.1 \%)$ \\
\hline & PHLF grade A & $6 / 83(7.2 \%)$ & $9 / 86(10.5 \%)$ \\
\hline & PHLF grade B & $3 / 83(3.6 \%)$ & $10 / 86(11.6 \%)$ \\
\hline & PHLF grade C & $0 / 83(0 \%)$ & $11 / 86(12.8 \%)$ \\
\hline \multirow[t]{4}{*}{ Ascites } & No or less than $500 \mathrm{ml} /$ day after POD 3 & $60 / 72(83.3 \%)$ & $36 / 74(48.7 \%)$ \\
\hline & Grade A (over 500 ml/day) & $8 / 72(11.1 \%)$ & $12 / 74(16.2 \%)$ \\
\hline & Grade B (requiring diuretics and/or albumin but less than $1000 \mathrm{ml} /$ day after POD 7) & $2 / 72(2.8 \%)$ & $11 / 74(14.8 \%)$ \\
\hline & Grade C (more than $1000 \mathrm{ml} /$ day after POD 7) & $2 / 72(2.8 \%)$ & $15 / 74(20.3 \%)$ \\
\hline \multirow[t]{5}{*}{ Hemorrhage } & No transfusion & $70 / 95(73.7 \%)$ & $66 / 96(68.7 \%)$ \\
\hline & Grade A & $8 / 95(8.4 \%)$ & $8 / 96(8.3 \%)$ \\
\hline & Grade B & $2 / 95(2.1 \%)$ & $7 / 96(7.3 \%)$ \\
\hline & Grade $\mathrm{C}$ & $3 / 95(3.2 \%)$ & $3 / 96(3.1 \%)$ \\
\hline & Reported as hemorrhage but without further detail & $12 / 95(12.6 \%)$ & $12 / 96(12.6 \%)$ \\
\hline \multirow[t]{5}{*}{ Infection complication } & No & $82 / 95(86.3 \%)$ & $54 / 96(56.2 \%)$ \\
\hline & Grade A (Clavien-Dindo grade II) & $9 / 95(9.5 \%)$ & $12 / 96(12.5 \%)$ \\
\hline & Grade B (Clavien-Dindo grade IIIa) & $3 / 95(3.2 \%)$ & $8 / 96(8.3 \%)$ \\
\hline & Grade C (Clavien-Dindo grade IIIb and more) & $1 / 95(1.0 \%)$ & $12 / 96(12.5 \%)$ \\
\hline & Reported as infection but without further detail & 0 & $10 / 96(10.5 \%)$ \\
\hline \multirow[t]{5}{*}{ Bile leaks } & No & $83 / 95(87.4 \%)$ & $67 / 97(69.1 \%)$ \\
\hline & Grade A & $9 / 95(9.5 \%)$ & $6 / 97(6.2 \%)$ \\
\hline & Grade B & $3 / 95(3.2 \%)$ & $7 / 97(7.2 \%)$ \\
\hline & Grade C & 0 & $7 / 97(7.2 \%)$ \\
\hline & Reported as bile leak but without further detail & 0 & $10 / 97(10.3 \%)$ \\
\hline
\end{tabular}

All the represented percentages in the Results Section have excluded the missing values

PHLF Post-hepatectomy liver failure

The causes of 90-day mortality were $\operatorname{PHLF}(n=10)$, infection complication $(n=4)$, biliary complication $(n=2)$, early tumor recurrence $(n=4)$, and other surgical complication (duodenum perforation, $n=1$ ).

\section{Risk Factors for Severe Complications (Clavien-Dindo Grades $3 b$ to 5)}

In the univariate analysis, statistically significant risk factors were age $>65$ years, intraoperative blood transfusion in stage-1 operation and FLR2/BW ratio $<$ $0.8 \%$. In multivariate analysis a FLR2/BW ratio $<0.8 \%$ was the only statistically significant risk factor (OR 2.92, $p=0.02$ ) for developing severe postoperative complications.

FLR2/sTLV $<40 \%$ or FLR2/BW ratio $<0.8 \%$ were found to be associated with PHLF by phi coefficient analysis with moderate negative relationships ( $\mathrm{phi}=0.307$ and 0.375 , respectively, $p<0.01$ ).
FIG. 1 Severe postoperative complications (Clavien-Dindo classification $3 \mathrm{~b}$ and 4 ) and 90-day mortality changes over years. The severe complication rate without 90 -day mortality was $60 \%$ in the early years and has since dropped to $29 \%$ recently. The 90-day mortality decreased from $40 \%$ to $7 \%$

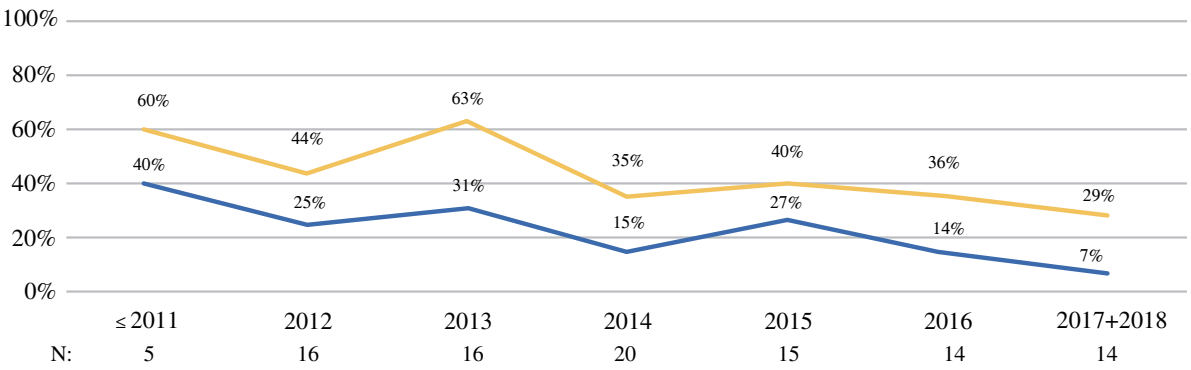

-90days mortality (y) CD classification $\geq 3 \mathrm{~b}$ 
Besides age $>65$ years, FLR2/sTLV $<40 \%$, FLR2/ $\mathrm{BW}<0.8 \%$ and elements of liver-surgery specific complications such as PHLF, ascites, infection, and PHH, but not bile leak, were associated with 90-day mortality by univariate logistic regression analysis. The multivariate analysis identified FLR2/sTLV $<40 \%$ as the only statistically significant risk factor for 90-day mortality (OR 5.52, $p=0.01$.

\section{Histopathological Results}

Among the studied population, adenocarcinoma was reported in $93.7 \%$ of cases (Table 3). Other subtypes of ICC were not specified. Positive lymph nodes were identified in $37.2 \%(n=35)$. Tumor multifocality was reported in $60 \%$. In non-tumor liver parenchyma, fibrosis was found in $26.2 \%$, cirrhosis in $2.4 \%$, and steatosis $>30 \%$ in $7.1 \%$ patients.

Based on the 7th edition of the AJCC staging system, the disease was classified as stage I in $7.1 \%$, stage II in $39.3 \%$, stage III in $11.9 \%$, and stage IVa in $41.7 \%$. A negative margin was achieved in 87 patients $(87.9 \%)$.

\section{Follow-Up}

The median follow-up by the reverse Kaplan-Meier method was 31.8 (17.4-46.2) months. Overall mortality was reported in $49.5 \%(n=49)$. The median OS was 26.4 months. The overall survival rate at $1,2,3$, and 5 years postoperatively was $64.3 \%, 52.5 \%, 38.8 \%$ and $22.0 \%$, respectively. At the end of follow-up, tumor recurrence (including intra- and extrahepatic recurrence) was reported in 53 patients $(55.8 \%)$. The median recurrence time was 9.3 months $(5.9-12.73)$. The overall recurrence rate at 1,2 , and 3 years postoperatively was $55.1 \%, 74.3 \%$, and $92 \%$, respectively. The intrahepatic recurrence rate at 1,2 , and 3 years postoperatively was $49.7 \%, 68 \%$, and $82.9 \%$, respectively. The extrahepatic recurrence rate at 1,2 , and 3 years postoperatively was $29.1 \%, 48.2 \%$, and $53.4 \%$, respectively.

According to preoperative imaging, a subgroup analysis of two groups of patients was performed: Group A with single lesion $(n=70)$; Group B with multiple lesions $(n=27)$. The 1-, 2-, and 3-year recurrence rates were $54.2 \%, 71.5 \%$, and $91.1 \%$ in Group A and $67.8 \%, 100 \%$, and $100 \%$ in Group B, with a median recurrence time of 11 (6.6-15.5) months in Group A and 4.5 (3.1-6) months in Group B $(p=0.03)$. Group B had a higher rate of extrahepatic recurrence than Group A (1-, 2-, and 3-year recurrence rates were $51.1 \%, 77.7 \%$, and $77.7 \%$ in Group B vs $22.6 \%, 39.4 \%$, and $46.1 \%$ in Group A, $p=0.01$ ) while the hepatic recurrence rate was similar in both groups.
TABLE 3 Histopathological features of 99 patients with intrahepatic cholangiocarcinoma (ICC)

\begin{tabular}{|c|c|c|}
\hline Variable & Definition & Patients $(n=99)$ \\
\hline \multirow[t]{2}{*}{ Histology } & Adenocarcinoma & 90/96 (93.7\%) \\
\hline & Other & $6 / 96(6.3 \%)$ \\
\hline \multirow[t]{2}{*}{ Margin } & Negative of tumor & $87 / 99(87.9 \%)$ \\
\hline & Positive of tumor & $12 / 99(12.1 \%)$ \\
\hline \multirow[t]{3}{*}{ Grading } & 1 & $7 / 87(6.9 \%)$ \\
\hline & 2 & $52 / 87(59.8 \%)$ \\
\hline & 3 & $29 / 87(33.3 \%)$ \\
\hline \multirow[t]{4}{*}{ Stage (AJCC 7th edition)* } & I & $6 / 84(7.1 \%)$ \\
\hline & II & $33 / 84(39.3 \%)$ \\
\hline & III & 10/84 (11.9\%) \\
\hline & IVa & $35 / 84(41.7 \%)$ \\
\hline \multirow[t]{6}{*}{ Primary tumor* } & $\mathrm{T} 1$ & $13 / 93(14 \%)$ \\
\hline & $\mathrm{T} 2$ & $38 / 93(40.9 \%)$ \\
\hline & $\mathrm{T} 2 \mathrm{a}$ & $6 / 93(6.4 \%)$ \\
\hline & $\mathrm{T} 2 \mathrm{~b}$ & $14 / 93(15 \%)$ \\
\hline & T3 & $20 / 93(21.5 \%)$ \\
\hline & $\mathrm{T} 4$ & $2 / 93(2.2 \%)$ \\
\hline \multirow[t]{2}{*}{ Nodal status* } & N0 & $59 / 94(62.8 \%)$ \\
\hline & N1 & $35 / 94(37.2 \%)$ \\
\hline \multirow[t]{2}{*}{ Metastasis* } & M0 & $94 / 94(100 \%)$ \\
\hline & M1 & 0 \\
\hline \multirow[t]{2}{*}{ Multifocal lesion } & $\mathrm{N}$ & $55 / 91(60.4 \%)$ \\
\hline & $\mathrm{Y}$ & $36 / 91(39.6 \%)$ \\
\hline Number of lesions & & $1(1-6)$ \\
\hline Largest tumor size $(\mathrm{mm})$ & & $85(6-260)$ \\
\hline Non-tumor & Normal & $51 / 84(60.7 \%)$ \\
\hline \multirow[t]{4}{*}{ Liver histology } & Fibrosis & $22 / 84(26.2 \%)$ \\
\hline & Steatosis $>30 \%$ & $6 / 84(7.1 \%)$ \\
\hline & CASH & $3 / 84(3.6 \%)$ \\
\hline & Cirrhosis & $1 / 84(2.4 \%)$ \\
\hline
\end{tabular}

All the represented percentages in the Results Section have excluded the missing values

*AJCC 7th edition of ICC was used for the TNM and tumor stage CASH Chemotherapy associated steatotic hepatitis, ICC Intrahepatic cholangiocarcinoma

\section{Propensity Score Matching Analysis}

Data with TNM staging from the AJCC 7th edition were only available between January 2010 and December 2013 in the SEER database. Within this time period, 453 patients with pathological confirmation of ICC, who received chemotherapy only, were identified.

After propensity score matching by age, gender, tumor stage and lymph node status, 88 patients in each group were included for further analysis. The median age was 65 years in the ALPPS group and 64 years in the chemotherapy (CTx) group. The stage distribution of 
tumors was stage I in $10.2 \%$, stage II in $38.6 \%$, stage III in $13.6 \%$, and stage IVa in $37.5 \%$ (Table 4 ).

The 90-day mortality in the ALPPS group was higher than in the CTx group (21.8\% vs $12.9 \%)$. Despite this, a superior OS was found in the ALPPS group with a median OS of 26.4 (12.6-40.2) months compared to 14 (11.4-16.6) months in the CTx group. The 1-, 2-, and 3-year survival rates were $66.0 \%, 55.9 \%$, and $40.1 \%$ in the ALPPS group and $52.6 \%, 14.1 \%$, and $11.3 \%$ in the CTx group, respectively ( $p<0.01$, Fig. 2).

By multivariate COX regression survival analyses, three statistically significant prognostic factors for poor overall survival were identified: (1) patients receiving chemotherapy only $(\mathrm{HR}=1.734, p=0.012)$; $(2)$ age $>65$ years $(\mathrm{HR}$ 1.561, $p=0.034$ ); and (3) lymph node metastasis (HR $1.653, p=0.022$ ).

As a FLR2/BW ratio less than $0.8 \%$ was the only significant risk factor for Clavien-Dindo grade $\geq 3 \mathrm{~b}$ complication in the multivariate analysis (OR 2.91, $p=0.02$ ), a subgroup analysis with FLR2/BW as the cutoff was analyzed. The benefit of ALPPS in regard to OS was confirmed in the subgroup with FLR2/BW ratio $\geq 0.8 \%(p<0.001)$ but not in the subgroup of FLR2/ $\mathrm{BW}<0.8 \%(p=0.231)$ compared to the CTx group (Fig. 3).

When the study population was divided according to preoperative imaging as Group A with a single lesion with the tumor centrally located and Group B with multiple lesions, the benefit of ALPPS on OS was confirmed in Group A $(p=0.004)$ but not in Group B $(p=0.247)$ when compared to the CTx group (Fig. 4).

\section{DISCUSSION}

Recently, ALPPS has been introduced as a novel solution that enables curative resection in marginally resectable tumors. ${ }^{34}$ Reports of the short-term outcomes of ALPPS in colorectal liver metastasis (CRLM), hepatocellular carcinoma (HCC), perihilar cholangiocarcinoma (CCA), but not ICC, have been published in the past. $^{20,23,35,36}$ A look into the current literature reveals that definitive evidence showing a benefit of the ALPPS procedure in terms of survival and oncological outcome in patients with ICC is still lacking. Additionally, there is no
TABLE 4 Propensity score matched patients of ALPPS group and chemotherapy (CTx) group

\begin{tabular}{|c|c|c|c|c|}
\hline & Definition & $\begin{array}{l}\text { CTx group } \\
(n=88)\end{array}$ & $\begin{array}{l}\text { ALPPS group } \\
(n=88)\end{array}$ & $p$ value \\
\hline \multirow[t]{3}{*}{ Age } & Continuous & $64(36-85)$ & $65(32-81)$ & 0.783 \\
\hline & $\leq 65$ & $45(51.1 \%)$ & $45(51.1 \%)$ & 1.000 \\
\hline & $>65$ & $43(48.9 \%)$ & $43(48.9 \%)$ & \\
\hline \multirow[t]{2}{*}{ Gender } & Female & $51(58 \%)$ & $51(58 \%)$ & 1.000 \\
\hline & Male & $37(42 \%)$ & $37(42 \%)$ & \\
\hline \multirow[t]{4}{*}{ Grade } & G1 & $5(8.8 \%)$ & $7(8.5 \%)$ & 0.908 \\
\hline & G2 & $32(56.4 \%)$ & $49(59.8 \%)$ & \\
\hline & G3 & $21(35.1 \%)$ & $26(31.7 \%)$ & \\
\hline & N.A. & $31(35.2 \%)$ & $4(5 \%)$ & \\
\hline \multirow[t]{4}{*}{ Stage* } & I & $9(10.2 \%)$ & $9(10.2 \%)$ & 1.000 \\
\hline & II & $34(38.6 \%)$ & $34(38.6 \%)$ & \\
\hline & III & $12(13.6 \%)$ & $12(13.6 \%)$ & \\
\hline & IVa & $33(37.5 \%)$ & $33(37.5 \%)$ & \\
\hline \multirow[t]{4}{*}{$\mathrm{T}^{*}$} & $\mathrm{~T} 1$ & $12(13.6 \%)$ & $12(13.6 \%)$ & 0.973 \\
\hline & $\mathrm{T} 2$ & $55(62.5 \%)$ & $55(62.5 \%)$ & \\
\hline & $\mathrm{T} 3$ & $18(20.5 \%)$ & $19(21.6 \%)$ & \\
\hline & $\mathrm{T} 4$ & $3(3.4 \%)$ & $2(2.3 \%)$ & \\
\hline \multirow[t]{2}{*}{$\mathrm{N}^{*}$} & N0 & $57(64.8 \%)$ & $57(64.8 \%)$ & 1.000 \\
\hline & N1 & $31(35.2 \%)$ & $31(35.2 \%)$ & \\
\hline \multirow[t]{2}{*}{$\mathrm{M}^{*}$} & M0 & $88(100 \%)$ & $88(100 \%)$ & 1.000 \\
\hline & M1 & $0-(0 \%)$ & $0-(0 \%)$ & \\
\hline Tumor Size (mm) & Continuous & $75(11-180)$ & $85(6-260)$ & 0.204 \\
\hline
\end{tabular}

*AJCC 7th edition was used for the TNM and tumor stage. All the represented percentages in the Results Section have excluded the missing values

ALPPS Associating liver partition and portal vein ligation for staged hepatectomy, CTx Chemotherapy 

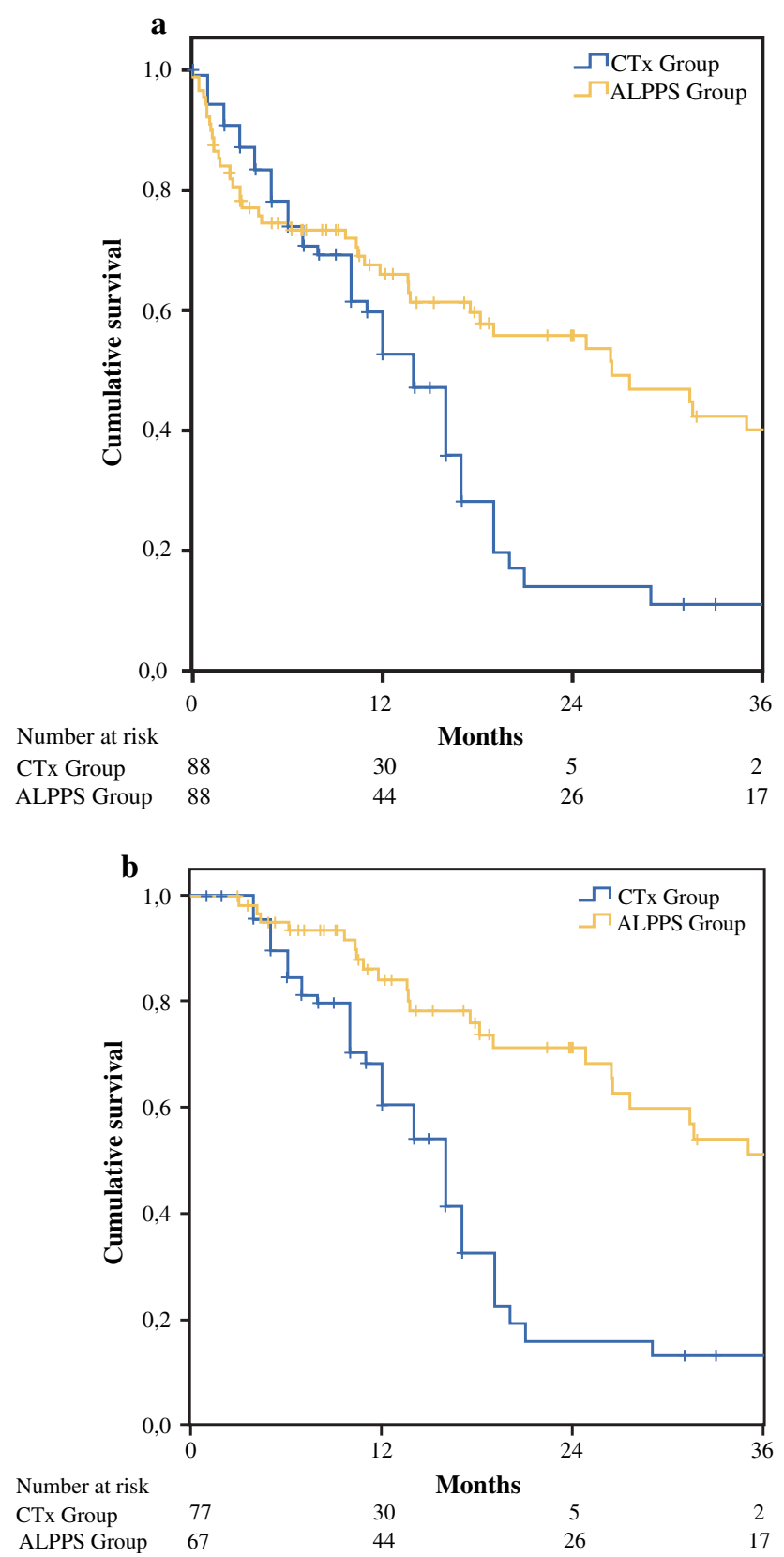

FIG. 2 Overall survival in ALPPS group and chemotherapy (CTx) group. A superior overall survival was found in the ALPPS group despite higher 90 -day mortality $(p<0.01)$. The difference in 2-year overall survival was $41.8 \%$ between the CTx group and the ALPPS group (a). The difference was $55.2 \%$ when 90 -day mortality was excluded (b)

large comparative study available to evaluate the riskbenefit balance of the ALPPS procedure in comparison to standard chemotherapy.

The results of our comparative survival analysis provide evidence for superior long-term outcomes of the ALPPS procedure over chemotherapy only. Despite the 90-day mortality, ALPPS shows superior 3-year overall survival rates compared to chemotherapy only $(39.6 \%$ vs $11.3 \%$,

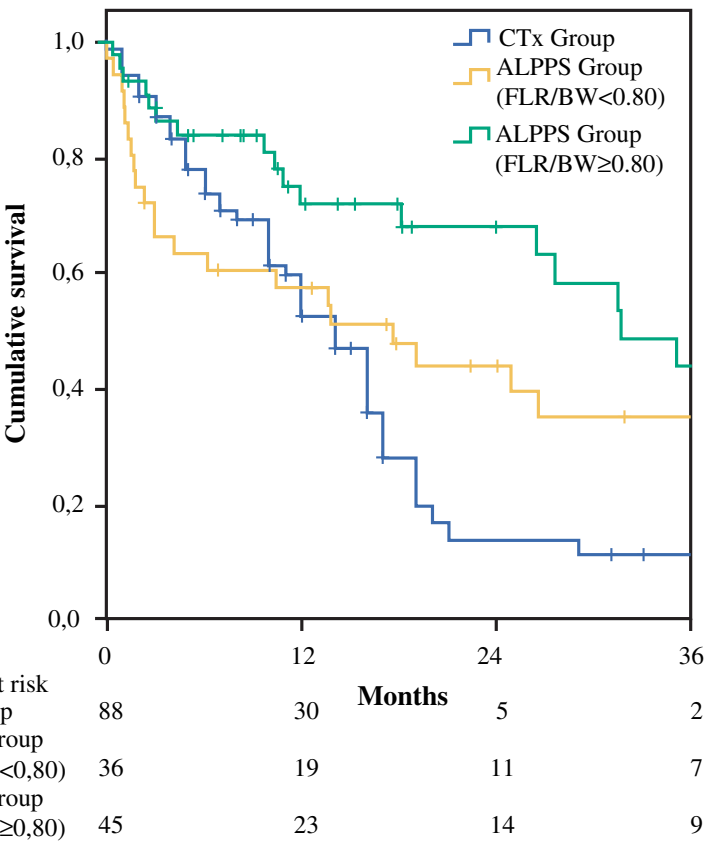

FIG. 3 Overall survival analysis according to FLR2/BW with $0.8 \%$ as the cutoff. The benefit of ALPPS on overall survival was confirmed in the subgroup with FLR2/BW ratio $\geq 0.8 \%(p<0.001)$ but not in FLR2/BW $<0.8 \%(p=0.231)$ compared to the CTx group

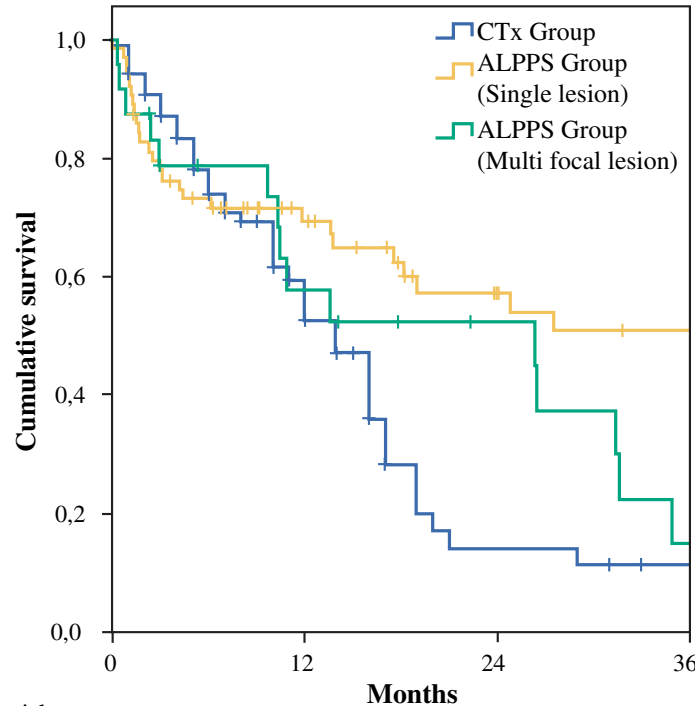

Number at risk CTx Group ALPPS Group (Single lesion) 88 30 5 2 ALPPS Group (Multi focal lesion) 24

33

19

15 11

7 2

FIG. 4 Overall survival analysis according to the number of lesions. The survival benefit of ALPPS over chemotherapy only can be found in patients with a single lesion (group $\mathrm{A}, p=0.004$ ) but not in patients with multiple lesions (group B, $p=0.247$ )

$p=0.01)$. The benefit of overall survival at 2 years was even greater, with a difference of $41.8 \%$ between the two groups (Fig. 2). Therefore, we believe that extensive liver 
resection by ALPPS procedure could provide better outcomes for patients with locally advanced ICC lesions, who are usually directed toward palliative chemotherapy. Longterm survival in this group of patients has also been achieved: the first ALPPS patient at the University Hospital Mainz celebrated her 10-year survival in August 2019. Except for an early intrahepatic recurrence during the 1st postoperative year, which was treated by repeated liver resection, the patient is now still disease free.

High morbidity and mortality rates after stage- 2 operations were observed in patients with ICC, comparable with morbidity and mortality rates following ALPPS for other primary liver malignancies, such as $\mathrm{HCC}$ or perihilar $\mathrm{CCA}^{20,23}$ The high complexity of surgery in this group of patients may be one of the main reasons for this observation. Indeed, trisectionectomy was performed in $78.7 \%$ of cases, biliary reconstruction in $35.6 \%$ and complex vascular reconstruction in $12.1 \%$. Liver surgery specific complications were reported as PHLF in $30.3 \%$, ascites in $49.3 \%$, bile leak in $30.3 \%$, infection complications in $42 \%$, and $\mathrm{PHH}$ in $35.3 \%$. Interestingly, when analyzing the risk factors for developing severe complications, lower future liver remnant volume at the stage-2 operation (FLR2), represented either by FLR2/BW $<0.8 \%$ or FLR2/ sTLV $<40 \%$, was the only statistically significant factor in the multivariate analysis. Moreover, FLR2/BW $<0.8 \%$ or FLR2/sTLV $<40 \%$, was found to be correlated with PHLF $(p<0.01)$. Till now there has been no recommendation for defining the threshold FLR volume heading into a stage-2 operation in ALPPS. A FLR/BW $\geq 0.5 \%$ in a non-cirrhotic liver is usually deemed as safe in one-stage hepatectomy. ${ }^{37}$ The cut-off of FLR/BW for a safe liver resection was indicated to be $0.8 \%$ in this study, much more liver volume than $0.5 \%$ in one-stage hepatectomy. It further supported the conclusion that liver volume does not necessarily equal liver function in the setting of ALPPS. ${ }^{38}$ Recently, 99mTc-mebrofenin hepatobiliary scintigraphy has been reported to be a valuable technique to estimate the risk of PHLF. ${ }^{39,40}$ In ALPPS, a liver function test has been applied in several institutes to ensure a safe outcome. ${ }^{41}$ However, a clear cut-off is still not available. Moreover, the liver function test by $99 \mathrm{mTc}$-mebrofenin hepatobiliary scintigraphy was not routinely performed at the majority of centers at the time of the current study. Based on the data on liver volume, the current study demonstrated that FLR2/ BW $<0.8 \%$ or FLR $2 / \mathrm{sTLV}<40 \%$ was a risk factor for PHLF and severe complications. Thus, a threshold of FLR2/BW as $0.8 \%$ or FLR2/sTLV as $40 \%$ is recommended in ICC patients waiting for stage-2 operation whenever liver function tests are not available.

The 90 -day mortality of $20.8 \%$ in our study is undisputedly high in comparison with mortality rates for conventional major hepatectomies or ALPPS performed for non-primary hepatobiliary malignancies. ${ }^{18,21,42} \mathrm{We}$ would like to emphasize that the reported results of early mortality in our cohort represent both initial and recent experiences. In 2017 and 2018, the 90-day mortality was $7 \%$ (1/14), which had dropped significantly from $40 \%$ before 2012. We therefore conclude that the learning curve and improved patient selection will eventually lead to acceptable mortality rates.

The high rate of severe postoperative complications also had a significant impact on the survival benefit of the ALPPS procedure over chemotherapy only. Among the propensity score-match (PSM) analysis, the 90-day mortality in the ALPPS group was higher than in the CTx group (21.6\% vs $12.5 \%)$. Despite this, a superior overall survival was found in the ALPPS group with a median survival time of 26.4 months compared to 14 months in the CTx group. Therefore, minimizing severe postoperative complications, especially the 90-day mortality, was among the most important tasks of ALPPS development. During recent years, major focus has been put on the surgical refinement of stage 1 operations. ${ }^{42} \mathrm{~A}$ variety of methods for liver partition have been proposed. ${ }^{17,43-45}$ Accordingly, postoperative morbidity and mortality rates have been reduced. ${ }^{42}$ However, the importance of sufficient FLR at stage-2 has not been previously emphasized. In the current study, we found that a small FLR at stage-2 was the only statistically significant risk factor regarding 90-day mortality. In PSM analysis, the benefit of ALPPS on overall survival was confirmed in the subgroup with FLR2/BW ratio $\geq 0.8 \%(p=0.001)$ but not in the subgroup of FLR2/ BW $<0.8 \%(p=0.294)$ in comparison to the chemotherapy only group. Indeed, achieving complete tumor resection (R0 in 87.9\%) in this study arguably led to higher postoperative morbidity and mortality rates. Decision making for the timing of stage-2 operations remains a major challenge, especially when quantitative liver function tests are not available. Caution should be taken when the FLR/BW ratio prior to stage- 2 operation is below $0.8 \%$. This may well be the most important lesson learned over the course of this study.

The risk for high postoperative morbidity and mortality rates in the ALPPS procedure with R0 resection should be weighed against alternative approaches. Until now, portal vein embolization (PVE) has been the mainstay for inducing FLR hypertrophy preoperatively in many institutions, whereas the superiority of ALPPS to PVE has been demonstrated in patients with CRLM. ${ }^{36}$ In the majority of cases in this cohort, PVE would have been technically very demanding or even impossible due to the tumor mass and location. A propensity score-match to compare ALPPS with PVE in this group of patients was unfortunately not feasible due to the small numbers in both groups. However, the choice of either ALPPS or PVE in the treatment of ICC 
needs to be investigated. Furthermore, the role of neoadjuvent chemotherapy with or without PVE in selected patients should be studied. Locoregional treatment strategies for advanced ICC include transarterial chemoembolization, yttrium-labeled selective internal radiation therapy, and hepatic arterial infusion. ${ }^{46,47}$ Despite promising results reported, the conclusive evidence for efficacy of these strategies is still lacking. ${ }^{1,8}$

In a large multi-institutional series of 301 patients with ICC, more than half of the patients experienced recurrence after resection. ${ }^{48}$ The majority of patients in that cohort had T1 category tumors $(58.1 \%)$. In the current cohort the patients suffered from more advanced tumor disease, with $53.4 \%$ showing AJCC stage III or IVa (T1 tumors were found only in $14 \%$; $60.2 \%$ patients had multiple lesions; lymph nodes metastases were found in $40.7 \%$ ). The advanced tumor stage led to a high tumor recurrence rate of $65.6 \%$ with a median recurrence time of 8 months. Intrahepatic recurrence was observed more frequently than extrahepatic recurrence. Patients with multiple lesions developed intrahepatic tumor recurrence earlier (4.5 vs 9 months in median) as well as more extrahepatic recurrence when compared to patients with a single lesion $(52.2 \%$ vs $23 \%)$. In a recent meta-analysis of 4756 patients, ICC with multiple lesions was confirmed as one of the most important factors predicting shorter overall survival. ${ }^{6}$ In the current study with PSM analysis, the benefit of ALPPS on overall survival was only confirmed in patients with single lesion, not in patients with multiple lesions, in comparison to systemic chemotherapy. Therefore, because of high postoperative morbidity and mortality rates as well as the limited benefit of adjuvant chemotherapy,${ }^{8}$ extensive liver resection using ALPPS could not be recommended as a better option than chemotherapy for ICC patients with multiple lesions. Since multiple lesions could represent systemic hematogenous metastatic intrahepatic dissemination or true multifocal disease, the prognostic relevance of them might be different. The former is definitely not an appropriate resection candidate.

The main limitation of this study is the retrospective methodology that leads to selection bias in both groups, especially that caused by under-reported cases with poor outcomes in patients undergoing ALPPS. The difference in experience of total cases of ALPPS per center as well as number of ICC patients might also have an influence on outcomes. Nevertheless, the use of propensity score matched analysis along with multivariate COX proportional hazard modeling has strengthened the degree of evidence and reduces this bias, and is more robustly precise than standard multivariable methods. The classifications used for the propensity score matching are $\mathrm{pT}$ and $\mathrm{pN}$ for the ALPPS group vs $\mathrm{cT}$ and $\mathrm{cN}$ for the chemotherapy-only group. This may underestimate the tumor stage in chemotherapy-only patients and lead to a negative selection bias. Moreover, comorbidity as well as Eastern Cooperative Oncology Group (ECOG) status was not included in the propensity score matching, which may lead to an overestimation of the overall survival in the ALPPS group. A randomized control trial to investigate short- and long-term outcomes would provide more reliable results.

We have utilized the AJCC staging system, 7th edition, in our analysis that was in line with the staging system utilized in both the ALPPS and SEER databases. Recently, the AJCC has released a new staging system for ICC in its 8th edition. Nevertheless, the 8th edition staging system for ICC has not shown a significant advantage over the 7 th edition in overall prognostic discrimination except for stage III, which represent only $8.7 \%$ of patients included in this study. ${ }^{49}$

From another aspect, a major advantage of this study was utilizing the ALPPS registry database as a baseline to create an international ALPPS prospective cohort with a longitudinal study design by retrieving new data on the ALPPS approach not reported by the registry web site. This advantage will facilitate the establishment of further studies to investigate long-term oncological outcomes of the ALPPS procedure and allow for risk adjustment analyses. Further work is needed to achieve significant improvements in regard to the quality of the data available for patients undergoing ALPPS. Therefore, we urge all surgeons performing this procedure to share their experiences and data through registration in the ALPPS registry (ww w.alpps.net).

In conclusion, this multicentric study demonstrated a high resectability of $97 \%$ and R0 resection of $85.3 \%$ in 102 patients with initially "unresectable" locally advanced ICC by ALPPS. Superior 1-, 2-, and 3-year overall survival rates were found in the ALPPS group compared with the chemotherapy only group, despite high 90-day mortality rates. Better patient selection and improved interstage decision making over time contributed to the decrease in mortality with time. PHLF was observed in $35 \%$ of the patients, with $12.8 \%$ classified as grade C. A threshold of FLR/BW of $0.8 \%$ (or $40 \%$ if FLR/sTLV is used) is recommended in patients waiting for the stage- 2 operation whenever liver function tests are not available. The benefit of ALPPS on overall survival was confirmed in ICC patients with a centrally located single lesion, but not for patients with multiple lesions when compared to chemotherapy only.

ACKNOWLEDGMENT Open Access funding provided by Projekt DEAL.

OPEN ACCESS This article is licensed under a Creative Commons Attribution 4.0 International License, which permits use, sharing, adaptation, distribution and reproduction in any medium or format, as 
long as you give appropriate credit to the original author(s) and the source, provide a link to the Creative Commons licence, and indicate if changes were made. The images or other third party material in this article are included in the article's Creative Commons licence, unless indicated otherwise in a credit line to the material. If material is not included in the article's Creative Commons licence and your intended use is not permitted by statutory regulation or exceeds the permitted use, you will need to obtain permission directly from the copyright holder. To view a copy of this licence, visit http://creativecommons. org/licenses/by/4.0/.

AUTHORS' CONTRIBUTIONS JL conceived and designed this study, acquired, analyzed and interpreted the data, made a first draft, revised, and gave final approval of the manuscript. All the authors made substantial contributions to acquisition of data, and analysis and interpretation of data. All the authors participated in drafting the article and revising it critically for important intellectual content. All the authors gave final approval of the version to be submitted.

\section{REFERENCES}

1. Banales JM, Cardinale V, Carpino G, et al. Expert consensus document: cholangiocarcinoma: current knowledge and future perspectives consensus statement from the European Network for the Study of Cholangiocarcinoma (ENS-CCA). Nat Rev Gastroenterol Hepatol. 2016;13(5):261-80.

2. Cardinale V, Semeraro R, Torrice A, et al. Intra-hepatic and extra-hepatic cholangiocarcinoma: new insight into epidemiology and risk factors. World $J$ Gastrointest Oncol. 15 2010;2(11):407-16.

3. Fitzmaurice C, Dicker D, et al. The global burden of cancer 2013. JAMA Oncol. 2015;1(4):505-527.

4. Benson AB, 3rd, D'Angelica MI, Abbott DE, et al. NCCN guidelines insights: hepatobiliary cancers, version 1.2017. J Natl Compr Canc Netw. 2017;15(5):563-73.

5. Bridgewater J, Galle PR, Khan SA, et al. Guidelines for the diagnosis and management of intrahepatic cholangiocarcinoma. $J$ Hepatol. 2014;60(6):1268-89.

6. Mavros MN, Economopoulos KP, Alexiou VG, Pawlik TM. Treatment and prognosis for patients with intrahepatic cholangiocarcinoma: systematic review and meta-analysis. JAMA Surg. 2014;149(6):565-74.

7. Valle J, Wasan $\mathrm{H}$, Palmer $\mathrm{DH}$, et al. Cisplatin plus gemcitabine versus gemcitabine for biliary tract cancer. $N$ Engl $J$ Med. 8 2010;362(14):1273-1281.

8. Weber SM, Ribero D, O'Reilly EM, Kokudo N, Miyazaki M, Pawlik TM. Intrahepatic cholangiocarcinoma: expert consensus statement. HPB (Oxford). 2015;17(8):669-80.

9. Khan SA, Davidson BR, Goldin RD, et al. Guidelines for the diagnosis and treatment of cholangiocarcinoma: an update. Gut. 2012;61(12):1657-1669.

10. Zhang XF, Beal EW, Bagante F, et al. Early versus late recurrence of intrahepatic cholangiocarcinoma after resection with curative intent. Br J Surg. 2018;105(7):848-56.

11. Farges O, Fuks D, Boleslawski E, et al. Influence of surgical margins on outcome in patients with intrahepatic cholangiocarcinoma: a multicenter study by the AFC-IHCC-2009 study group. Ann Surg. 2011;254(5):824-9; discussion 830.

12. Ribero D, Pinna AD, Guglielmi A, et al. Surgical approach for long-term survival of patients with intrahepatic cholangiocarcinoma: a multi-institutional analysis of 434 patients. Arch Surg. 2012;147(12):1107-13.
13. Tamandl D, Herberger B, Gruenberger B, Puhalla H, Klinger M, Gruenberger T. Influence of hepatic resection margin on recurrence and survival in intrahepatic cholangiocarcinoma. Ann Surg Oncol. 2008;15(10):2787-94.

14. de Santibanes E, Clavien PA. Playing Play-Doh to prevent postoperative liver failure: the "ALPPS" approach. Ann Surg. 2012;255(3):415-7.

15. Schnitzbauer AA, Lang SA, Goessmann H, et al. Right portal vein ligation combined with in situ splitting induces rapid left lateral liver lobe hypertrophy enabling 2-staged extended right hepatic resection in small-for-size settings. Ann Surg. 2012;255(3):405-14.

16. Alvarez FA, Ardiles V, de Santibanes M, Pekolj J, de Santibanes E. Associating liver partition and portal vein ligation for staged hepatectomy offers high oncological feasibility with adequate patient safety: a prospective study at a single center. Ann Surg. 2015;261(4):723-32.

17. Li J, Ewald F, Gulati A, Nashan B. Associating liver partition and portal vein ligation for staged hepatectomy: from technical evolution to oncological benefit. World J Gastrointest Surg. 27 2016;8(2):124-33.

18. Schadde E, Ardiles V, Robles-Campos R, et al. Early survival and safety of ALPPS: first report of the International ALPPS Registry. Ann Surg. 2014;260(5):829-36; discussion 836-828.

19. Schadde E, Malago M, Hernandez-Alejandro $R$, et al. Monosegment ALPPS hepatectomy: extending resectability by rapid hypertrophy. Surgery. 2015;157(4):676-89.

20. D'Haese JG, Neumann J, Weniger M, et al. Should ALPPS be used for liver resection in intermediate-stage HCC? Ann Surg Oncol. 2016;23(4):1335-1343.

21. Hernandez-Alejandro R, Bertens KA, Pineda-Solis K, Croome KP. Can we improve the morbidity and mortality associated with the associating liver partition with portal vein ligation for staged hepatectomy (ALPPS) procedure in the management of colorectal liver metastases? Surgery. 2015;157(2):194-201.

22. Li J, Girotti P, Konigsrainer I, Ladurner R, Konigsrainer A, Nadalin S. ALPPS in right trisectionectomy: a safe procedure to avoid postoperative liver failure? $J$ Gastrointest Surg. 2013;17(5):956-61.

23. Olthof PB, Coelen RJS, Wiggers JK, et al. High mortality after ALPPS for perihilar cholangiocarcinoma: case-control analysis including the first series from the international ALPPS registry. HPB (Oxford). 2017;19(5):381-7.

24. Vauthey JN, Abdalla EK, Doherty DA, et al. Body surface area and body weight predict total liver volume in Western adults. Liver Transpl. 2002;8(3):233-40.

25. Kambakamba P, Stocker D, Reiner CS, et al. Liver kinetic growth rate predicts postoperative liver failure after ALPPS. HPB (Oxford). 2016;18(10):800-5.

26. van den Broek MA, van Dam RM, van Breukelen GJ, et al. Development of a composite endpoint for randomized controlled trials in liver surgery. Br J Surg. 2011;98(8):1138-45.

27. Li J, Moustafa M, Freiwald-Bibiza E, Alzudjali A, Fischer L, Nashan B. Is it feasible to standardize a composite postoperative complication reporting system for liver resection? J Gastrointest Surg. 2019. https://doi.org/10.1007/s11605-019-04457-w.

28. Dindo D, Demartines N, Clavien PA. Classification of surgical complications: a new proposal with evaluation in a cohort of 6336 patients and results of a survey. Ann Surg. 2004;240(2):205-13.

29. Koch M, Garden OJ, Padbury R, et al. Bile leakage after hepatobiliary and pancreatic surgery: a definition and grading of severity by the International Study Group of Liver Surgery. Surgery. 2011;149(5):680-8. 
30. Rahbari NN, Garden OJ, Padbury R, et al. Posthepatectomy liver failure: a definition and grading by the International Study Group of Liver Surgery (ISGLS). Surgery. 2011;149(5):713-24.

31. Rahbari NN, Garden OJ, Padbury R, et al. Post-hepatectomy haemorrhage: a definition and grading by the International Study Group of Liver Surgery (ISGLS). HPB (Oxford). 2011;13(8):528-35.

32. Clavien PA, Petrowsky H, DeOliveira ML, Graf R. Strategies for safer liver surgery and partial liver transplantation. $N$ Engl J Med. 12 2007;356(15):1545-59.

33. Li J, Kantas A, Ittrich H, et al. Avoid "all-touch" by hybrid ALPPS to achieve oncological efficacy. Ann Surg. 2016;263(1):e6-7.

34. Schadde E, Ardiles V, Slankamenac K, et al. ALPPS offers a better chance of complete resection in patients with primarily unresectable liver tumors compared with conventional-staged hepatectomies: results of a multicenter analysis. World J Surg. 2014;38(6):1510-9.

35. Lang H, de Santibanes E, Schlitt HJ, et al. 10th Anniversary of ALPPS-lessons learned and quo vadis. Ann Surg. 2019;269(1):114-9.

36. Sandstrom P, Rosok BI, Sparrelid E, et al. ALPPS improves resectability compared with conventional two-stage hepatectomy in patients with advanced colorectal liver metastasis: results from a Scandinavian multicenter randomized controlled trial (LIGRO trial). Ann Surg. 2018;267(5):833-40.

37. Truant S, Oberlin O, Sergent G, et al. Remnant liver volume to body weight ratio $>$ or $=0.5 \%$ : A new cut-off to estimate postoperative risks after extended resection in noncirrhotic liver. $J \mathrm{Am}$ Coll Surg. 2007;204(1):22-33.

38. Tanaka K, Matsuo K, Murakami T, et al. Associating liver partition and portal vein ligation for staged hepatectomy (ALPPS): short-term outcome, functional changes in the future liver remnant, and tumor growth activity. Eur $J$ Surg Oncol. 2015;41(4):506-12.

39. de Graaf W, van Lienden KP, Dinant S, et al. Assessment of future remnant liver function using hepatobiliary scintigraphy in patients undergoing major liver resection. J Gastrointest Surg. 2010;14(2):369-78.

40. de Graaf W, van Lienden KP, van Gulik TM, Bennink RJ. $(99 \mathrm{~m}) \mathrm{Tc}-\mathrm{mebrofenin}$ hepatobiliary scintigraphy with SPECT for the assessment of hepatic function and liver functional volume before partial hepatectomy. J Nucl Med. 2010;51(2):229-36.

41. Serenari M, Collaud C, Alvarez FA, et al. Interstage assessment of remnant liver function in ALPPS using hepatobiliary scintigraphy: prediction of posthepatectomy liver failure and introduction of the HIBA index. Ann Surg. 2018;267(6):1141-7.

42. Linecker M, Bjornsson B, Stavrou GA, et al. Risk adjustment in ALPPS is associated with a dramatic decrease in early mortality and morbidity. Ann Surg. 2017;266(5):779-86.

43. Edmondson MJ, Sodergren MH, Pucher PH, et al. Variations and adaptations of associated liver partition and portal vein ligation for staged hepatectomy (ALPPS): many routes to the summit. Surgery. 2016;159(4):1058-72.

44. de Santibanes E, Alvarez FA, Ardiles V, Pekolj J, de Santibanes M. Inverting the ALPPS paradigm by minimizing first stage impact: the mini-ALPPS technique. Langenbecks Arch Surg. 2016;401(4):557-63.

45. Sakamoto Y, Matsumura M, Yamashita S, Ohkura N, Hasegawa K, Kokudo N. Partial TIPE ALPPS for perihilar cancer. Ann Surg. 2018;267(2):e18-20.

46. Hoffmann RT, Paprottka PM, Schon A, et al. Transarterial hepatic yttrium-90 radioembolization in patients with unresectable intrahepatic cholangiocarcinoma: factors associated with prolonged survival. Cardiovasc Intervent Radiol. 2012;35(1):105-16.

47. Hyder O, Marsh JW, Salem R, et al. Intra-arterial therapy for advanced intrahepatic cholangiocarcinoma: a multi-institutional analysis. Ann Surg Oncol. 2013;20(12):3779-86.

48. Hyder O, Hatzaras I, Sotiropoulos GC, et al. Recurrence after operative management of intrahepatic cholangiocarcinoma. Surgery. 2013;153(6):811-8.

49. Spolverato G, Bagante F, Weiss M, et al. Comparative performances of the 7th and the 8th editions of the American Joint Committee on Cancer staging systems for intrahepatic cholangiocarcinoma. J Surg Oncol. 2017;115(6):696-703.

Publisher's Note Springer Nature remains neutral with regard to jurisdictional claims in published maps and institutional affiliations. 